\title{
The Epsilon Algorithm and Operational Formulas of Numerical Analysis
}

\author{
By P. Wynn
}

1. Introduction. It is the purpose of this paper to describe a non-linear technique which appears to have powerful and general application in numerical analysis. However, before doing so it is necessary to refer to a few related theoretical concepts.

2. Rational Operational Formulas. The double sequence of rational functions

$$
\frac{U_{\mu, \nu}(x)}{V_{\mu, \nu}(x)}
$$$$
\mu, \nu=0,1, \cdots
$$

where

$$
\frac{U_{\mu, \nu}(x)}{V_{\mu, \nu}(x)}=\frac{\alpha_{\mu, \nu, 0}+\alpha_{\mu, v, 1} x+\cdots+\alpha_{\mu, \nu, \nu} x^{\nu}}{\beta_{\mu, y, 0}+\beta_{\mu, \nu, 1} x+\cdots+\beta_{\mu, \nu, \mu} x^{\mu}}
$$

may be derived from the series

$$
\beta(x)=\sum_{s=0}^{\infty} c_{s} x^{2}
$$

by imposing the condition that the power series expansion of (1) should agree with (2) as far as the term in $x^{\mu+\nu}$. If none of the Hankel determinants

$$
\left|\begin{array}{cccc}
c_{m} & c_{m+1} & \cdots & c_{m+k-1} \\
c_{m+1} & c_{m+2} & \cdots & c_{m+k} \\
\vdots & \vdots & & \vdots \\
c_{m+k-1} & c_{m+k} & \cdots & c_{m+2 k-2}
\end{array}\right| \quad m, k-1=0,1, \cdots
$$

vanish, and the additional condition $\beta_{k, v, 0}=1$ is imposed, the coefficients in the rational expression (1) are uniquely determined. The rational expressions (1) may be placed in a two-dimensional array in which the quotient (1) occurs at the intersection of the $(\mu+1)$ th row and the $(\nu+1)$ th column. [1] [2] [3].

As is well known, the numerical convergence of the sequence

$$
\frac{U_{r, r}(x)}{V_{r, r}(x)}
$$

$$
r=0,1, \cdots
$$

for a particular value of $x$ is in many cases much better than that of the series (2). This consideration led Kopal [4] to the consideration of rational operational formalas, that is, to the replacement of the operational equation

$$
\left(\sum_{s=0}^{\infty} c_{s} d^{e}\right) F=f
$$

where $F$ is a known function from which $f$ is to be determined, and $d$ is a finite dis-

Received November 1959; revised June 1960. 
placement operator, by the equation

$$
\left(\frac{U_{r, r}(d)}{V_{r, r}(d)}\right) F=f
$$

Equation (5) cannot at the moment, in its non-linear form, be solved. The equation may however be linearized by multiplication throughout by $V_{r, r}(d)$ to give

$$
U_{r, r}(d) F=V_{r, r}(d) f .
$$

Assuming that $F$ and $f$ are completely known, that $r$ in equation (6) is sufficiently large, and the example is a suitable one, then there will exist considerable numerical agreement between the right and left hand sides of equation (6). Assuming that $d$ is any one of the conventional operators $\Delta, E, \nabla, \mu, \delta$ of numerical analysis, and that $F$ and the sequences of values $f_{1}, f_{2}, \cdots ; f_{-1}, f_{-2}, \cdots$ are known, then equation (6) may be rearranged so as to determine $f_{0}$. It is this very last assumption which constitutes a serious limitation of the linearizing technique resulting in equation (6). Indeed, Kopal was only able to find useful application of the technique when $d$ was the backward difference operator, though his numerical results, which related to the forward integration of a differential equation, appeared to be very promising. However, the same effect over a very much larger range of problems may be achieved by recourse to another method.

3. The $e_{m}\left(S_{n}\right)$ Transformation. In his researches into the non-linear transformation*

$$
e_{m}\left(S_{n}\right)=\frac{\left|\begin{array}{cccc}
S_{n} & S_{n+1} & \cdots & S_{n+m} \\
\Delta S_{n} & \Delta S_{n+1} & \cdots & \Delta S_{n+m} \\
\vdots & \vdots & \cdots & \vdots \\
\Delta S_{n+m-1} & \Delta S_{n+m} & \cdots & \Delta S_{n+2 m-1}
\end{array}\right|}{\left|\begin{array}{cccc}
1 & 1 & \cdots & 1 \\
\Delta S_{n} & \Delta S_{n+1} & \cdots & \Delta S_{n+m} \\
\vdots & \vdots & \cdots & \vdots \\
\Delta S_{n+m-1} & \Delta S_{n+m} & \cdots & \Delta S_{n+2 m-1}
\end{array}\right|}
$$

$$
m, n=0,1, \cdots
$$

of the sequence $S_{r}, r=0,1, \cdots$ Shanks [5], by an appeal to the theory of linear equations, showed that if

$$
S_{r}=\sum_{s=0}^{r} c_{s} x^{s} \quad r=0,1, \cdots
$$

then

$$
e_{m}\left(S_{n}\right)=\frac{U_{m, m+n}(x)}{V_{m, m+n}(x)}
$$$$
m, n=0,1, \cdots .
$$

The same result may be derived from the theory of orthogonal polynomials [6].

4. The e-Algorithm. The evaluation of the determinants in the various expressions (7) is sufficiently laborious to be prohibitive. However, the expressions (7)

* The notation used here is consistent with that of [7] but differs slightly from that of [5] where the right hand side of (7) would be designated as $e_{m}\left(S_{n+m}\right)$. 
may be computed recursively by means of the $\epsilon$ Algorithm as follows [7]. If, from the initial conditions

$$
\epsilon_{1}^{(m)}=0 \quad m=1,2, \cdots ; \quad \epsilon_{0}^{(m)}=S_{m} \quad m=0,1, \ldots
$$

quantities $\epsilon_{z}^{(m)}$ are computed recursively using the relation

$$
\epsilon_{s+1}^{(m)}=\epsilon_{s-1}^{(m+1)}+\frac{1}{\epsilon_{s}^{(m+1)}-\epsilon_{s}^{(m)}} \quad m, s=0,1, \cdots,
$$

then

$$
\epsilon_{2 s+1}^{(m)}=\left\{e_{s}\left(\Delta S_{m}\right)\right\}^{-1} \quad \epsilon_{2 s}^{(m)}=e_{s}\left(S_{m}\right) \quad m, s=0,1, \cdots .
$$

If the quantities $\epsilon_{s}^{(m)}$ are arranged in the scheme

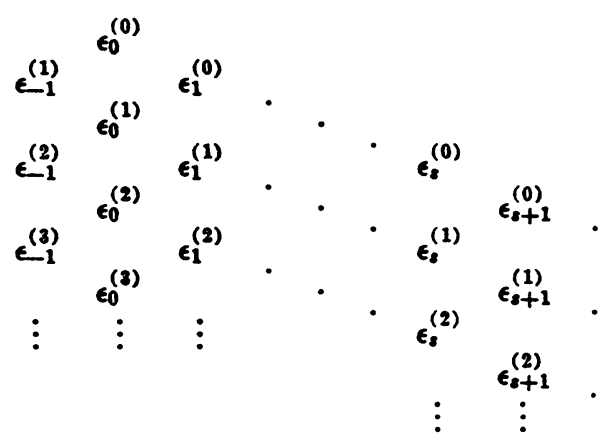

it will be seen that relations (11) may be used, column by column, to build up the scheme from left to right. It should be noted that if conformity, by means of equations (9) and (12), is to take place between the Pade Table and the $\epsilon$-array, the latter must be transposed about the diagonal $m=0$; the columns of the $\epsilon$-array with even order suffixes then take their place as rows in the Pade Table.

The following theorem, based upon the results of the last two sections, may now be given:

ThEOREM. If $p$ is an associative and commutative operator, and

$$
a_{s} p^{s} F=c_{s} x^{s}
$$$$
s=0,1, \cdots
$$

and quantities $\epsilon_{s}^{(m)}$ are computed using the relation (11) from the initial values

$$
\epsilon_{-1}^{(m)}=0 \quad m=1,2, \cdots ; \quad \epsilon_{0}^{(m)}=\sum_{s=0}^{m} a_{s} p^{s} F
$$

then

$$
\epsilon_{2 s}^{(m)}=\frac{U_{s, m+s}(x)}{V_{s, m+s}(x)} \quad m, s=0,1, \cdots
$$

First ExaMple: A numerical example of the application of the theorem now follows. It concerns the process of obtaining the derivative at $z=0$ of the function $\exp (h z)$, when $h=0.6$, by means of the formula

$$
\left(\frac{d}{d z} F\right)_{s=0}=\sum_{s=0}^{\infty} \frac{(-1)^{s}}{s+1} \Delta^{s+1} F
$$


Here, in the notation of equation (13)

$$
\frac{(-1)^{s}}{(s+1)} \Delta^{s+1} F=\frac{(-1)^{s}}{(s+1)}\left(e^{h}-1\right)^{s+1} \text {. }
$$

The quantities with even order suffix in the $\epsilon$-array for this example are displayed in Table I.

Note: The results of Table I begin with the diagonal $m=1$. If the notation of equation (15) is strictly to be adhered to, an entry $\epsilon_{0}^{(0)}=0$ together with a corresponding diagonal should be appended to Table I. However, this is not a matter of great importance, and in the event that an operational series were to begin with a term in $p^{s}, s>1$, even this artifice would not be available.

It is perhaps. in order to comment upon the power of the algorithm as revealed by this example. Attainment of the same accuracy as is achieved in Table I by the straightforward use of the series (16), even neglecting the accumulation of round-off errors, would involve the summation of about eighty terms and an excursion into arithmetic involving twenty-eight decimal figures.

The Padé quotients ( 3 ) in this example are successive convergents of the continued fraction

$$
x^{-1} \log (1+x)=\frac{1}{1+} \frac{1^{2} x}{2+} \frac{1^{2} x}{3+} \frac{2^{2} x}{4+} \frac{2^{2} x}{5+} \cdots .
$$

Numerical investigation into the behavior of this continued fraction [8] shows that application of the $\epsilon$-algorithm to the series (16) converges quite reasonably for $\left(e^{h z}-1\right)>1$, when the series rapidly diverges.

In the derivation of the classical operational formulas of numerical analysis the operand is assumed to be a polynomial, and the formulas derived are then completely valid. The formulas are then universally applied, without examination of the operand, and without any more justification than that of the results achieved.

In the same way it occurs that although formula (13) is no longer valid, use of the $\epsilon$-algorithm in conjunction with operational series meets with success. Two examples which support this thesis now follow.

Secord Example: This concerns the interpolation of the function $\log (0.6+h z)$ when $h=0.1$ and $z=0.25$ with points of tabulation at unit intervals of $z$, by use

TABLE I

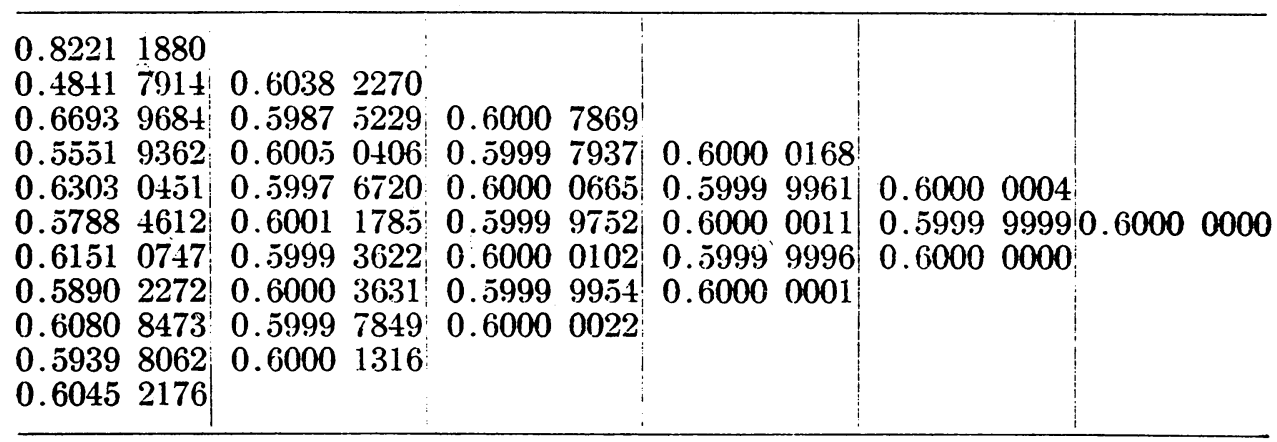


of Bessel's Interpolation Formula

$$
F(z)=\sum_{s=0}^{\infty} a_{s} F(0)
$$

where

$$
\begin{aligned}
a_{0} & =1 \quad a_{1}=2 \delta E^{1 / 2} \\
a_{2 s} & =\left(\begin{array}{c}
2+s-1 \\
2 s
\end{array}\right) \mu \delta^{2 s} E^{1 / 2} \\
a_{2 s+1} & =\frac{z-\frac{1}{2}}{2 s+1}\left(\begin{array}{c}
2+s-1 \\
2 s
\end{array}\right) \delta^{2 s+1} E^{1 / 2} s=1,2, \cdots
\end{aligned}
$$

The quantities $\epsilon_{s}^{(m)}$ with even order suffix are displayed in Table II.

Since $\log (0.625)=-0.4700036$, it will be seen that application of the $\epsilon$-algorithm results in an effective gain of three decimal figures. This is not spectacular, but there is no point in selecting for presentation only those examples which display the method in a particularly favorable light. It might be mentioned at this point that the author has experimented with the $\epsilon$-algorithm in conjunction with operational formulas in a large number of cases, and in none of these was the accuracy of the transformed results worse than the original partial sums.

Since the odd and even order terms in the series (19) are so dissimilar, the odd and even terms were separated out and the two series submitted separately to treatment by the $\epsilon$-algorithm, the transformed results subsequently being added together. The numerical results produced in this way were not, however, significantly better than those shown in Table II.

Third Example: This concerns the application of the Euler-Maclaurin integration formula

$$
\int_{x}^{x+h} F(t)=d t=\frac{h}{2}\{F(x)+F(x+h)\}-\sum_{s=1}^{m} \frac{h^{2 s+1} B_{2 s}}{(2 s) !} \Delta_{h} F^{(2 s-1)}(x)
$$

when the integrand is the function $\exp \left(-z^{2}\right)$ and the upper and lower limits of integration are 0 and $w(1+i)$ respectively, with $w=0.75$.

\begin{tabular}{|c|c|c|c|c|}
\hline $\begin{array}{rr}-0.4337 & 503 \\
0.4722 & 880 \\
0.4700 & 009 \\
0.4699 & 419 \\
0.4700 & 084 \\
0.4700 & 105 \\
0.4700 & 020 \\
0.4700 & 017 \\
-0.4700 & 048\end{array}$ & $\begin{array}{rr}-0.4701 & 290 \\
0.4699 & 404 \\
0.4699 & 732 \\
0.4700 & 105 \\
0.4700 & 088 \\
0.4700 & 017 \\
-0.4700 & 019\end{array}$ & 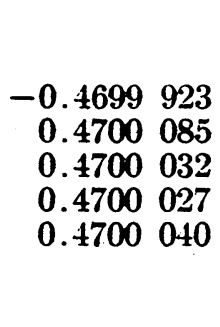 & $\begin{array}{r}-0.4700040 \\
0.4700027 \\
-0.4700031\end{array}$ & -0.4700034 \\
\hline
\end{tabular}

The functions $u_{s}=\frac{h^{s+2} F^{(s)}(h)}{(s+1) !}$ in this example satisfy the recursion

$$
s(s+1) u_{s}+2 h^{2} s u_{s-1}+2 h^{2}(s-1) u_{s-2}=0
$$

TABLE II 


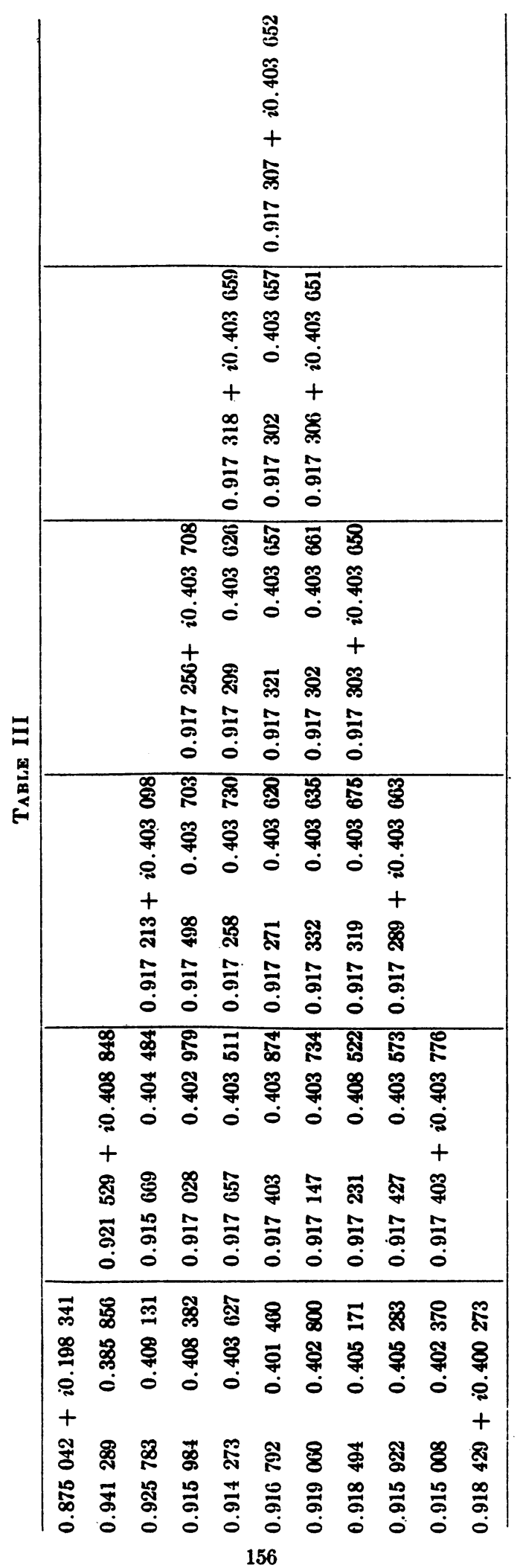


with

$$
\begin{aligned}
& u_{0}=-w\left\{\cos \left(2 w^{2}\right)+\sin \left(2 w^{2}\right)\right\}-i w\left\{\cos \left(2 w^{2}\right)-\sin \left(2 w^{2}\right)\right\} \\
& u_{1}=-2 w^{3}\left\{\cos \left(2 w^{2}\right)-\sin \left(2 w^{2}\right)\right\}+i 2 w^{3}\left\{\cos \left(2 w^{2}\right)+\sin \left(2 w^{2}\right)\right\} .
\end{aligned}
$$

The quantities $\epsilon_{*}^{(m)}$ (which are now complex numbers) with even order suffix are displayed in Table III. Since erf $(0.75(1+i))=0.917306+i 0.403654$, application of the $\epsilon$-algorithm has in this case resulted in the gain of about three decimal places.

It is perhaps of interest to point out that the accuracy of the transformed results produced in the first and third examples could have been increased by extending the computation. This is also true to a limited extent of the second example, but the non-existence of central differences above a certain order limits the extent to which the computation may be prolonged.

It would be useful, when examining the mathematical validity of the procedures adopted in the second and third examples, to be able to relate the determinantal quotient

$$
\begin{array}{|cccc}
\sum_{s=0}^{s-0} c_{s} p^{s} F & \sum_{s=0}^{s-1} c_{s} p^{s} F & \cdots & \sum_{s=0}^{s-m} c_{s} p^{s} F \\
c_{1} p F & c_{2} p^{2} F & \cdots & c_{m+1} p^{m+1} F \\
\vdots & \vdots & & \vdots \\
c_{m} p^{m} F & c_{m+1} p^{m+1} F & \cdots & c_{2 m} p^{2 m} F \\
\hline 1 & 1 & \cdots & 1 \\
c_{1} p F & c_{2} p^{2} F & \cdots & c_{m+1} p^{m+1} F \\
\vdots & \vdots & & \vdots \\
c_{m} p^{m} F & c_{m+1} p^{m+1} F & \ldots & c_{2 m} p^{2 m} F
\end{array}
$$

to the solution $f$ of the operational equation

$$
\sum_{s=0}^{\infty} c_{s} p^{s} F=f
$$

but this appears to be one of the cases in which a statement of the problem is not a great step forward to its solution.

5. Acknowledgments. This paper was written when the author was a member of the Istitut für Angewandte Mathematik in the University of Mainz; he is grateful to the Deutsche Forschungsgemeinschaft for a research grant which enabled the work to be carried out. The numerical results given were produced on the Z-22 at at Mainz.

\section{Mathematisch Centrum} Amsterdam

1. H. PADE, "Sur la Representation Approchée d'une Fonction par des Fractions Rationelles," Ann. Ec. Norm. Sup. 3, 1892, p. 9 .

2. O. Perron, Die Lehre von den Kettenbrüchen, Chelsea, New York, 1950, p. 420. 377.

3. H. WALL, Analytic Theory of Continued Fractions, van Nostrand, New York, 1948, p. 
4. Z. KOPAL, "Operational methods in numerical analysis based on rational approximations," On Numerical Approximation, University of Wisconsin Press, Madison, 1959, p. 25.

5. D. SHaNks, "Non-linear transformation of divergent and slowly convergent sequences," Jn. Math. and Phys., v. 3t, 1955, p. 21

6. P. WYNN, "The rational approximation of functions which are formally defined by a power series expansion," Math. Comp., v. 14, 1960, p. 147.

7. P. WrN , "On a device for computing the $e_{m}\left(S_{n}\right)$ transformation," $M T A C$, v. 10, 1956, p. 91 .

8. P. WYNN, "The numerical efficiency of certain continued fraction expansions," to appear. 